\title{
More on generalized simplicial chiral models
}

\author{
M. Alimohammadi ${ }^{a, b}$ 円, Kh. Saaidi ${ }^{a}$ Z \\ ${ }^{a}$ Physics Department, University of Tehran, North Karegar Ave., \\ Tehran, Iran \\ ${ }^{b}$ Institute for Studies in Theoretical Physics and Mathematics, \\ P.O.Box 5531, Tehran 19395, Iran
}

\begin{abstract}
By generalizing the auxiliary field term in the Lagrangian of simplicial chiral models on a $(d-1)$-dimensional simplex, the generalized simplicial chiral models has been introduced in [1]. These models can be solved analytically only in $d=0$ and $d=2$ cases at large $-\mathrm{N}$ limit. In $d=0$ case, we calculate the eigenvalue density function in strong regime and show that the partition function computed from this density function is consistent with one calculated by path integration directly. In $d=2$ case, it is shown that all $V=\operatorname{Tr}\left(A A^{\dagger}\right)^{n}$ models have a third order phase transition, same as the 2-dimensional Yang-Mills theory.
\end{abstract}

\footnotetext{
${ }^{1} \mathrm{e}-$ mail:alimohmd@theory.ipm.ac.ir

${ }^{2}$ e-maill:lkhled@molavi.ut.ac.ir
} 


\section{Introduction}

The $1 / \mathrm{N}$ expansion of matrix-valued field theories is probably the most important nonperturbative and nonnumerical theoretical tool presently available in the study of such models as non-Abelian gauge theories and two-dimensional quantum gravity. The first major result concerning the large- $\mathrm{N}$ limit of matrix theories was due to 't Hooft, who made the crucial observation that in the $1 / \mathrm{N}$ expansion of continuum gauge theories, the set of Feynman diagrams contributing to any given order admits a simple topological interpretation [2].

Unfortunately, our knowledge of exact solution for the large $-\mathrm{N}$ limit is limited to a small number of few-matrix systems, and it becomes even smaller if we restrict ourselves to the case of unitary matrix fields, which is relevant to the problem of lattice QCD. After the solution of Gross and Witten [3] of the single link problem and its generalizations, exact results were obtained only for the external field problem [ [4, 5] and a few toy models ( $\mathrm{L}=3,4$ chiral chains) [6, 7]. Therefore extending the number of solved few-matrix systems is an important progress from different points of view: first from pure theoretical reason. Second by noticing that any few-matrix system have a reinterpretation, via double scaling limit, as some different kind of matter coupled to two-dimensional quantum gravity. And third because every few-matrix system involving unitary matrices can be reinterpreted as the generating functional for a class of integrals over unitary groups, and these integrals in turn are the essential missing ingredient in the context of a complete algorithmization of the strong coupling expansion of many interesting models [8].

One of the interesting classes of the few-matrix models is the simplicial chiral model (SCM) [9]. In this model, each unitary matrix interacts in a fully symmetric way with all other matrices, by preserving the global chiral invariance. The resulting system can be described as a chiral model on $(d-1)$-dimensional simplex. A simplex is formed by connecting $d$ vertices by $d(d-1) / 2$ links. In ref. [10], where the large-N saddle-point equations for density function $\rho(z)$ have been found, the authors have introduced a single auxiliary variable $A$ (a complex matrix) to decouple the matrix interaction, and by performing the single-link external field integral, the saddle-point equation in strong and weak regions have been found. In $d=2$, $d=4$, and $d \rightarrow \infty$, the saddle-point equation has been solved analytically and the phase transition of the model has been specified. In the action of SCM in terms of the matrix $A$, this matrix appears as a $\operatorname{Tr}\left(A A^{\dagger}\right)$ term.

On the other hand, it is well known that the pure 2-dimensional Yang-Mills theory $\left(\mathrm{YM}_{2}\right)$ can be represented by the Lagrangian $i \operatorname{Tr}(B F)+\operatorname{Tr}\left(B^{2}\right)$, in which $F$ is the usual field-strength tensor and $B$ is an auxiliary pseudo-scalar field. Many of the main properties of $\mathrm{YM}_{2}$ does not change if one considers the generalized two-dimensional $\mathrm{YM}_{2}\left(\mathrm{gYM}_{2}\right)$,

which can be found by replacing $\operatorname{Tr}\left(B^{2}\right)$ term in $\mathrm{YM}_{2}$ action by an arbitrary class function $f(B)[11$. The partition function of these theories have been fully studied in different contexts 
in refs.[1] - [13] and the phase structure of some of the specific examples has been studied in refs. 14] and 15. In all the studied cases, it is seen that the models have third-order phase transition, which is the same behavior as $\mathrm{YM}_{2}$. The crucial point in this area is that such investigation for continuum $\mathrm{gYM}_{2}$ is very complicated and there is no general result for the phase transition of an arbitrary $\mathrm{gYM}_{2}$ theory.

In ref. [1], the procedure used to obtain the $\mathrm{gYM}_{2}$ from $\mathrm{YM}_{2}$, i.e. $\operatorname{Tr}\left(B^{2}\right) \rightarrow f(B)$, has been used in SCM to introduce the generalized simplicial chiral model (gSCM). That is,

the $\operatorname{Tr}\left(A A^{\dagger}\right)$ term in SCM has been replaced by an arbitrary class function $V\left(A A^{\dagger}\right)$. The large- $\mathrm{N}$ limit of the model has been discussed and the saddle-point equations in strong and weak regimes have been found. Note that as the SCM at $d=2$ is the discrete version of $\mathrm{YM}_{2}$ [10], we can consider the gSCM, at $d=2$, as the equivalent matrix theory of $\mathrm{gYM}_{2}$. The phase structure of the model for $d=2$ and $V=\operatorname{Tr}\left(A A^{\dagger}\right)^{n}(n=2,3,4)$ is also obtained in [1].

In this paper we want to complete our investigation of $d=2$ gSCM and show that all $V=\operatorname{Tr}\left(A A^{\dagger}\right)^{n}$ models (with $n$ an arbitrary positive integer) have third-order phase transition. We think that it is an important result as it indirectly proves the equivalence of all $\operatorname{Tr}\left(B^{n}\right) \mathrm{gYM}_{2}$ theories with $\mathrm{YM}_{2}$, from the phase structure point of view. The plane of the paper is as follows. In section 2, we bring a brief review of SCM and gSCM and also the saddle-point equations in weak and strong regimes. In section 3 , we focus on $d=0$ case and show that the calculation of the partition function of the model, described in terms of $A$ fields, leads to the trivial result which obtained from the main action. This is a consistency check of our procedure. Finally in section 4 we investigate the phase structure of $V=\operatorname{Tr}\left(A A^{\dagger}\right)^{n}$ gSCM in $d=2$ and show that all of these models have third-order phase transition. We also discuss the variation of the discontinuity of $\beta^{2} \partial C(\beta, N) / \partial \beta$ with $n$ at the critical point $\left(C\right.$ is the specific heat and $\beta=\left(g^{2} N\right)^{-1}$, where $g$ is the coupling constant).

\section{The gSCM}

If we assign a $\mathrm{U}(N)$ matrix to each vertex of a $(d-1)$-dimensional simplex, then the partition function of simplicial chiral models is defined by [9]

$$
Z_{d}(\beta, N)=\int \prod_{i=1}^{d} d U_{i} \exp \left\{N \beta \sum_{i=1}^{d} \sum_{j=i+1}^{d} \operatorname{Tr}\left(U_{i} U_{j}^{\dagger}+U_{i}^{\dagger} U_{j}\right)\right\}
$$

To find this partition function, the authors of [9] have introduced a single auxiliary variable $A$ to decouple the matrix interactions. The resulting partition function is

$$
Z_{d}=\tilde{Z}_{d} / \tilde{Z}_{0}
$$


where

$$
\tilde{Z}_{d}=\int \prod_{i=1}^{d} d U_{i} d A \exp \left\{-N \beta \operatorname{Tr} A A^{\dagger}+N \beta \operatorname{Tr} A \sum_{i} U_{i}^{\dagger}+N \beta \operatorname{Tr} A^{\dagger} \sum_{i} U_{i}-N^{2} \beta d\right\} .
$$

Performing the single-link integral over the matrices $U_{i}$

$$
e^{N W\left(B B^{\dagger}\right)} \equiv \int d U \exp \left[N \operatorname{Tr}\left(B^{\dagger} U+U^{\dagger} B\right)\right]
$$

we obtain

$$
\tilde{Z}_{d}=\int d A \exp \left\{-N \beta \operatorname{Tr} A A^{\dagger}+N d W\left(\beta^{2} A A^{\dagger}\right)-N^{2} \beta d\right\} .
$$

The gSCM is defined through the action [1]

$$
Z_{d, V}=\tilde{Z}_{d, V} / \tilde{Z}_{0, V}
$$

in which

$$
\tilde{Z}_{d, V}=\int d A \exp \left\{-N \beta V\left(A A^{\dagger}\right)+N d W\left(\beta^{2} A A^{\dagger}\right)-N^{2} \beta d\right\},
$$

where $V\left(A A^{\dagger}\right)$ is an arbitrary class function of $A A^{\dagger}$ :

$$
V\left(A A^{\dagger}\right)=\sum_{n=1} a_{n} \operatorname{Tr}\left(A A^{\dagger}\right)^{n}
$$

In special case $\alpha_{n}=\delta_{n, 1}$, the gSCM reduces to SCM. If we denote the eigenvalues of the Hermitian semi-positive-definite matrix $\beta^{2} A A^{\dagger}$ by $x_{i}$ 's, and at the large- $\mathrm{N}$ limit, which we

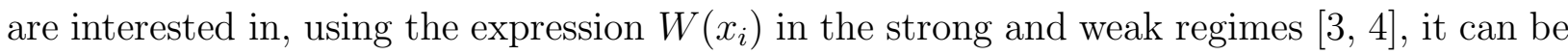
shown that the saddle-point equation for the eigenvalue density function $\rho(z)\left(z_{i}=\sqrt{x_{i}+c}\right)$ is [1]:

$$
z \sum_{k=1} \frac{k a_{k}}{\beta^{2 k-1}}\left(z^{2}-c\right)^{k-1}-d=\frac{1}{2} \mathcal{P} \int_{a}^{b} d z^{\prime} \rho\left(z^{\prime}\right)\left(\frac{2}{z-z^{\prime}}-\frac{d-2}{z+z^{\prime}}\right) .
$$

In the above equation $\mathcal{P}$ indicates the principal value of integral, and the parameters $a$ and $b$ must be determined dynamically. The normalization condition of $\rho(z)$ is

$$
\int_{a}^{b} \rho\left(z^{\prime}\right) d z^{\prime}=1
$$

In the weak coupling regime $\left(\beta>\beta_{c}\right), c_{w}=0$ and the density function $\rho(z)$ must satisfy

$$
\int_{a}^{b} d z^{\prime} \frac{\rho\left(z^{\prime}\right)}{z^{\prime}} \leq 2
$$

In the strong coupling regime $\left(\beta<\beta_{c}\right), c_{s}=a^{2}$ and $\rho(z)$ must satisfy

$$
\int_{a}^{b} d z^{\prime} \frac{\rho\left(z^{\prime}\right)}{z^{\prime}}=2 .
$$


Using the standard method of solving the integral equation [16], it can be shown that the density function $\rho(z)$ in weak regime satisfies the following equation

$$
\begin{aligned}
& \rho_{w}(z)=\frac{\sqrt{(b-z)(z-a)}}{\pi}\left\{\sum_{m, p, q} \frac{m a_{m}}{\beta^{2 m-1}} C_{p} C_{2 m-p-q-2} z^{q} a^{p} b^{2 m-p-q-2}\right. \\
& \left.-\frac{d-2}{2} \int_{a}^{b} \frac{d y}{y+z} \frac{\rho_{w}(y)}{\sqrt{(b+y)(y+a)}}\right\}, \quad \text { for } \beta>\beta_{c}
\end{aligned}
$$

where $C_{n}=(2 n-1) ! ! /\left(2^{n} n !\right)$, and in strong regime satisfies

$$
\begin{aligned}
& \rho_{s}(z)=-\frac{z}{\pi} \sqrt{\frac{b-z}{z-a}}\left\{\sum_{n, m, p, q} \frac{n a_{n}}{\beta^{2 n-1}}\left(-a^{2}\right)^{p}\left(\begin{array}{c}
n-1 \\
p
\end{array}\right) B_{m} C_{2 n-2 p-m-q-2} z^{q} a^{m} b^{2 n-2 p-m-q-2}\right. \\
& \left.+\frac{d-2}{2} \int_{a}^{b} \frac{d y}{y+z} \sqrt{\frac{y+a}{y+b}} \frac{\rho_{s}(y)}{y}\right\}, \quad \text { for } \beta<\beta_{c},
\end{aligned}
$$

where $B_{m}=(2 m-3) ! ! /\left(2^{m} m !\right)\left(B_{0} \equiv-1\right)$ [1]. Also by investigating the behavior of the integrals at $z \rightarrow \infty$, it can be shown that the parameters $a$ and $b$ in $\beta>\beta_{c}$ must be determined from the following equations

$$
\sum_{n, m} \frac{n a_{n}}{\beta^{2 n-1}} C_{m} C_{2 n-m-1} a^{m} b^{2 n-m-1}-2=0
$$

and

$$
\sum_{n, m} \frac{n a_{n}}{\beta^{2 n-1}} C_{m} C_{2 n-m} a^{m} b^{2 n-m}-(a+b)=1,
$$

and in strong regime, $\beta<\beta_{c}$, from the equations

$$
\sum_{n, m, p} \frac{n a_{n}}{\beta^{2 n-1}}\left(-a^{2}\right)^{p}\left(\begin{array}{c}
n-1 \\
p
\end{array}\right) B_{m} C_{2 n-2 p-m-1} a^{m} b^{2 n-2 p-m-1}+2=0,
$$

and

$$
-\sum_{n, m, p} \frac{n a_{n}}{\beta^{2 n-1}}\left(-a^{2}\right)^{p}\left(\begin{array}{c}
n-1 \\
p
\end{array}\right) B_{m} C_{2 n-2 p-m} a^{m} b^{2 n-2 p-m}+a-b=1 .
$$

Finally if we denote the internal energy per unit link by $U_{d, V}$, we have by definition

$$
\frac{d(d-1)}{2} U_{d, V}=\frac{1}{2 N^{2}} \frac{\partial}{\partial \beta} \ln Z_{d, V}(\beta, N) .
$$

After some calculation, it can be shown that at large- $\mathrm{N}$ limit, the internal energies in weak and strong regimes for $V=\operatorname{Tr}\left(A A^{\dagger}\right)^{n}$ are:

$$
d(d-1) U_{d, n}^{(w)}=\frac{2 n-1}{\beta^{2 n}} \int_{a}^{b} z^{2 n} \rho_{w}(z) d z-d+\left(\frac{1}{n}-2\right) \frac{1}{\beta},
$$

and

$$
d(d-1) U_{d, n}^{(s)}=\frac{2 n-1}{\beta^{2 n}} \int_{a}^{b}\left(z^{2}-a^{2}\right)^{n} \rho_{s}(z) d z-d+\left(\frac{1}{n}-2\right) \frac{1}{\beta} .
$$

These equations can be used to deduce the order of phase transition of the models. 


\section{$3 \quad d=0$ gSCM with $V=\operatorname{Tr}\left(A A^{\dagger}\right)^{n}$}

It is clear from the definition of $Z_{d, V}$ in eq.(6) that $Z_{0, V}=1$, however $\tilde{Z}_{0, V}$ is not trivial. To see this, let us focus on $V=\operatorname{Tr}\left(A A^{\dagger}\right)^{n}$ from now on. It is not difficult to show that $\tilde{Z}_{0, n}$ from eq.(7) is [1]

$$
\tilde{Z}_{0, n} \sim \exp \left[\frac{N^{2}(2 n-1)}{2} \ln \beta\right] .
$$

Now to check the procedure introduced in the last section for studying the theory in the large $-\mathrm{N}$ limit, it is instructive to reproduce this result by using the density function $\rho(z)$ at $d=0$.

At $d=0$, the saddle-point equation (9) in weak regime $(c=0)$, for the case $a_{k}=\delta_{k, n}$, becomes

$$
\frac{n}{2 \beta^{2 n-1}} z^{2 n-2}=\mathcal{P} \int_{a}^{b} \frac{\rho_{w}\left(z^{\prime}\right)}{z^{2}-z^{2}} d z^{\prime} \quad, \quad \beta>\beta_{c}
$$

and in the strong regime $\left(c=a^{2}\right)$ becomes

$$
\frac{n}{2 \beta^{2 n-1}}\left(z^{2}-a^{2}\right)^{n-1}=\mathcal{P} \int_{a}^{b} \frac{\rho_{s}\left(z^{\prime}\right)}{z^{2}-z^{2}} d z^{\prime} \quad, \quad \beta<\beta_{c} .
$$

Let us focus on $\beta<\beta_{c}$ case. To make the integral equation (24) more conventional, we use the change of variable $z \rightarrow \lambda=z^{2}$, with density function $\rho_{s}(\lambda)$ satisfies

$$
\rho_{s}(\lambda) d \lambda=\rho_{s}\left(z^{\prime}\right) d z^{\prime}
$$

So

$$
\frac{n}{2 \beta^{2 n-1}}\left(\lambda-a^{2}\right)^{n-1}=\mathcal{P} \int_{a^{2}}^{b^{2}} \frac{\rho_{s}(\xi)}{\lambda-\xi} d \xi \quad, \quad \beta<\beta_{c} .
$$

Now consider the function $H_{s}(\lambda)$ in complex $-\lambda$ plane

$$
H_{s}(\lambda)=\int_{a^{2}}^{b^{2}} \frac{\rho_{s}(\xi)}{\lambda-\xi} d \xi
$$

This function is analytic on the entire complex plane except for a cut on the positive real axis in the interval $\left[a^{2}, b^{2}\right]$. Then one has

$$
H_{s}(\lambda \pm i \epsilon)=R_{s}(\lambda) \mp i \pi \rho_{s}(\lambda) \quad, \quad a^{2} \leq \lambda \leq b^{2}
$$

where $R_{s}(\lambda)$ is, from eq.(26),

$$
R_{s}(\lambda)=\frac{n}{2 \beta^{2 n-1}}\left(\lambda-a^{2}\right)^{n-1} .
$$

Using the standard method of solving the integral equations [16], one can show that the expression

$$
H_{s}(\lambda)=\frac{1}{2 \pi i} \sqrt{\frac{\lambda-b^{2}}{\lambda-a^{2}}} \oint_{c} \sqrt{\frac{\xi-a^{2}}{\xi-b^{2}}} \frac{R_{s}(\xi)}{\lambda-\xi} d \xi
$$


has the correct analytical behavior in $\beta<\beta_{c}$ region (see [1] for more details). Here the contour $c$ is a contour encircling the cut $\left[a^{2}, b^{2}\right]$ and excluding $\lambda$. Deforming $c$ to a contour around the point $\lambda$ and the contour $c_{\infty}$ (a contour at infinity), one finds

$$
H_{s}(\lambda)=R_{s}(\lambda)+\frac{1}{2 \pi i} \sqrt{\frac{\lambda-b^{2}}{\lambda-a^{2}}} \oint_{c_{\infty}} \sqrt{\frac{\xi-a^{2}}{\xi-b^{2}}} \frac{R_{s}(\xi)}{\lambda-\xi} d \xi
$$

Inserting (29) in (31), it can be easily shown that $\rho_{s}(\lambda)$ is (using (28)):

$$
\rho_{s}^{(n)}(\lambda)=-\frac{n}{2 \pi \beta^{2 n-1}} \sqrt{\frac{b^{2}-\lambda}{\lambda-a^{2}}} \sum_{m, p, s=0}(-1)^{s}\left(\begin{array}{c}
n-1 \\
s
\end{array}\right) B_{p} C_{n-p-m-s-1} \lambda^{m} a^{2(p+s)} b^{2(n-p-m-s-1)} .
$$

At $n=1$, where gSCM reduces to SCM, it can be shown that (32) is equal

$$
\rho_{s}^{n=1}(\lambda)=\frac{1}{2 \pi \beta} \sqrt{\frac{b^{2}-\lambda}{\lambda-a^{2}}}
$$

which is the same as one calculated in [10]. To find the parameters $a$ and $b$ in eq.(32), we note that at $|\lambda| \rightarrow \infty$, eqs. (27) and (10) imply $H(\lambda) \rightarrow 1 / \lambda$ or $\sqrt{\left(\lambda-a^{2}\right) /\left(\lambda-b^{2}\right)} H(\lambda) \rightarrow 1 / \lambda$. Therefore we can expand $\sqrt{\left(\lambda-a^{2}\right) /\left(\lambda-b^{2}\right)}\left(R_{s}(\lambda)-i \pi \rho_{s}(\lambda)\right)$ and take the coefficients of $\lambda^{0}$ and $1 / \lambda$ equal to 0 and 1 , respectively. It can be see that the coefficient of $\lambda^{0}$ is identically zero, and the second condition yields to (for $\beta<\beta_{c}$ )

$$
\frac{n}{2 \beta^{2 n-1}} \sum_{p, s=0}(-1)^{s}\left(\begin{array}{c}
n-1 \\
s
\end{array}\right) B_{p} C_{n-s-p} a^{2(p+s)} b^{2(n-p-s)}=1 .
$$

In $n=1$ case, eq.(34) reduces to $b^{2}-a^{2}=4 \beta$, which is one obtained in [10]. The same calculation for the density function $\rho_{w}(\lambda)$ yields to

$$
\rho_{w}^{(n)}(\lambda)=\left\{\begin{array}{lc}
0 & n=1 \\
\frac{n}{2 \pi \beta^{2 n-1}} \sqrt{\left(b^{2}-\lambda\right)\left(\lambda-a^{2}\right)} \sum_{p, s=0} C_{p} C_{n-p-s-2} \lambda^{s} a^{2 p} b^{2(n-p-s-2)} & n>1
\end{array}\right.
$$

and the following equations which specify the parameters $a$ and $b$ in $\beta>\beta_{c}$,

$$
\begin{gathered}
\frac{n}{2 \beta^{2 n-1}} \sum_{p=0} C_{p} C_{n-p-1} a^{2 p} b^{2(n-p-1)}=0, \\
\frac{n}{2 \beta^{2 n-1}} \sum_{p=0} C_{p} C_{n-p} a^{2 p} b^{2(n-p)}=1 .
\end{gathered}
$$

Now let us focus on critical point $\beta=\beta_{c}$ in which $a=a_{c}$ and $b=b_{c}$ must satisfy eqs.(34), (36) and (37) simultanously. It is not difficult to see at $\beta=\beta_{c}$, except the first term of eqs. (34) and (37) which is equal, all the other terms are not the same. The only unique solution of this inconsistency is

$$
a_{c}=0
$$


Inserting (38) in eqs.(36) and (37) (or (34)), results

$$
\begin{gathered}
\frac{b_{c}^{2 n-2}}{\beta_{c}^{2 n-1}}=0, \\
\frac{n}{2 \beta_{c}^{2 n-1}} C_{n} b_{c}^{2 n}=1 .
\end{gathered}
$$

The solution of these equations are

$$
\begin{aligned}
& b_{c} \longrightarrow \infty, \\
& \beta_{c} \longrightarrow \infty,
\end{aligned}
$$

such that eq. (40) holds. Now in the weak-coupling region, where $\rho_{w} \sim 1 / \beta^{2 n-1}, \beta$ is always greater than $\beta_{c}=\infty$, so $\rho_{w} \rightarrow 0$. Therefore there is only one regime at $d=0$, i.e. the strong-coupling regime.

To check eq.(22), it is easier first to calculate the contribution of $\tilde{Z}_{0, n}$ to internal energy $U_{0, n}^{(s)}$ and then find $\tilde{Z}_{0, n}$ from it. If we denote this contribution by $\tilde{U}_{0, n}^{(s)}$, it is equal to (see eq. (21))

$$
\begin{aligned}
\tilde{U}_{0, n}^{(s)} & =\frac{2 n-1}{2 \beta^{2 n}} \int_{a}^{b}\left(z^{2}-a^{2}\right)^{n} \rho_{s}^{(n)}(z) d z \\
& =\frac{2 n-1}{2 \beta^{2 n}} \int_{a^{2}}^{b^{2}}\left(\lambda-a^{2}\right)^{n} \rho_{s}^{(n)}(\lambda) d \lambda
\end{aligned}
$$

in which $\rho_{s}^{(n)}(\lambda)$ is given by eq.(32) and the relation between $a$ and $b$ can be obtained from (34). To see the consistency of the results, it is sufficient to check some specific examples. In $n=2$, (42) reduces to

$$
\tilde{U}_{0,2}^{(s)}=\frac{27}{256 \beta^{7}}\left(b^{2}-a^{2}\right)^{4}
$$

and (34) reduces to

$$
\frac{3}{8}\left(b^{2}-a^{2}\right)^{2}=\beta^{3}
$$

therefore

$$
\tilde{U}_{0,2}^{(s)}=\frac{3}{4 \beta}=\frac{1}{2 N^{2}} \frac{\partial}{\partial \beta} \ln \tilde{Z}_{0,2}
$$

This equation gives $\tilde{Z}_{0,2} \sim \exp \left(\frac{3 N^{2}}{2} \ln \beta\right)$, which is the same behavior as eq.(22). It can be also shown that for $n=3,4$, and 5 , these two methods have the same result. As another reason for the fact that only the strong-coupling regime exists in $d=0$, it can be seen that the $\tilde{Z}_{0, n}$ calculated from $\rho_{w}(\lambda)$ (eq.(35)) does not coincide with eq.(22). 


\section{Phase structure of gSCM at $d=2$}

As mentioned earlier, the gSCM can be solved analytically only in $d=0$ and $d=2$ cases, which the latter is an important case because of its equivalence to $\mathrm{gYM}_{2}$ (and in special $\left.\mathrm{YM}_{2}\right)$. So in this section we want to study the gSCM at $d=2$ for $V=\operatorname{Tr}\left(A A^{\dagger}\right)^{n}$.

It is clear from eqs.(13) and (14) that the density function are known in the weak and strong regimes at $d=2$, as the second term in the right-hand sides of these equations vanishes. It can also be shown that at $d=2, \rho_{w}(0)$ must be equal to zero at critical point $\beta=\beta_{c}$. In this way, one can obtain the precise value of $a, b$, and $\beta$ at critical point as following [四:

$$
\begin{aligned}
a_{c} & =0 \\
b_{c} & =\frac{2 n}{2 n-1} \\
\beta_{c} & =\frac{2 n}{2 n-1}\left[\frac{n(4 n-3) ! !}{2^{2 n}(2 n-1) !}\right]^{\frac{1}{2 n-1}} .
\end{aligned}
$$

To study the phase structure of this model, it is necessary to calculate the internal energies in both weak and strong regimes. We know the functional form of the density function in these two regimes, but there are two unknown parameters $a$ and $b$ in each of these densities which must be obtained from the eqs.(15) and (16) for weak regime and from eqs.(17) and (18) for strong regime. Unfortunately these equations are too complicated to be solved exactly, but the crucial point is that as we want to study the phase structure of the model, it is sufficient to look at the solutions near the critical point. Therefore we expand the equations (15) and (16) around $a_{c}=0$ and $b_{c}=2 n /(2 n-1)$, and find $a_{w}$ and $b_{w}$ in terms of $\alpha=\beta-\beta_{c}$ up to second order. After a lengthy calculation one finds

$$
\begin{aligned}
a_{w} & =\frac{4 n-3}{2(2 n-1) \beta_{c}} \alpha-\frac{4 n-3}{2 n(4 n-5) \beta_{c}^{2}} \alpha^{2}+\ldots \\
b_{w} & =\frac{2 n}{2 n-1}+\frac{4 n-1}{2(2 n-1) \beta_{c}} \alpha-\frac{1}{8 n \beta_{c}^{2}} \alpha^{2}+\ldots
\end{aligned}
$$

Now inserting $\rho_{w}(z)$ from eq.(13), for $a_{m}=\delta_{n, m}$ and $d=2$, into eq.(20), we arrive at

$$
U_{2, n}^{(w)}=-\frac{n(2 n-1)}{2 \pi \beta^{4 n-1}} \sum_{p, q, r=0} B_{r} C_{p} C_{2 n-p-q-2} a^{p+r} b^{4 n-p-q-2} I(2 n+q-r)-1+\left(\frac{1}{2 n}-1\right) \frac{1}{\beta},
$$

in which

$$
I(k)=\frac{\sqrt{\pi} b^{2+k} \Gamma(3 / 2+k)}{2 \Gamma(3+k)}-\frac{a^{\frac{3}{2}+k} \sqrt{b}}{\frac{3}{2}+k}{ }_{2} F_{1}\left[-\frac{1}{2}, \frac{3}{2}+k, \frac{5}{2}+k, \frac{a}{b}\right],
$$

where ${ }_{2} F_{1}[a, b, c, x]$ is the hypergeometric function. Keeping the terms up to second order of $a_{w}^{2}$, eq.(48) becomes:

$$
U_{2, n}^{(w)}=K\left(T_{1} b_{w}^{4 n}-\frac{1}{2} T_{2} b_{w}^{4 n-1} a_{w}+T_{3} b_{w}^{4 n-2} a_{w}^{2}+\ldots\right)-1+\left(\frac{1}{2 n}-1\right) \frac{1}{\beta},
$$


where

$$
\begin{gathered}
K=\frac{n(2 n-1)}{4 \sqrt{\pi} \beta^{4 n-1}}, \\
T_{1}=\sum_{q=0} C_{2 n-q-2} M(n, q, 0), \\
T_{2}=C_{2 n-2} M(n, 0,1), \\
T_{3}=\sum_{q=0}\left[\frac{3}{8} C_{2 n-q-4} M(n, q, 0)-\frac{1}{4} C_{2 n-q-3} M(n, q, 1)-\frac{1}{8} C_{2 n-q-2} M(n, q, 2)\right],
\end{gathered}
$$

in which

$$
M(n, q, r)=\frac{\Gamma(3 / 2+2 n+q-r)}{\Gamma(3+2 n+q-r)} .
$$

In the strong regime, the same calculation leads to

$$
\begin{aligned}
a_{s} & =-\frac{4 n-3}{2(2 n-1) \beta_{c}} \alpha-\frac{(n-1)(4 n-3)}{2 n(4 n-5) \beta_{c}^{2}} \alpha^{2}+\cdots, \\
b_{s} & =\frac{2 n}{2 n-1}+\frac{4 n-1}{2(2 n-1) \beta_{c}} \alpha+\frac{n-1}{2 n \beta_{c}^{2}} \alpha^{2}+\cdots,
\end{aligned}
$$

for the parameters $a$ and $b$, and

$$
U_{2, n}^{(s)}=K\left(T_{1} b_{s}^{4 n}+\frac{1}{2} T_{2} b_{s}^{4 n-1} a_{s}+S_{3} b_{s}^{4 n-2} a_{s}^{2}+\ldots\right)-1+\left(\frac{1}{2 n}-1\right) \frac{1}{\beta},
$$

for internal energy. $S_{3}$ in eq.(54) is

$$
S_{3}=\sum_{q=0}\left[\left(\frac{7}{8}-n\right) C_{2 n-q-4} M(n, q, 0)-\frac{1}{4} C_{2 n-q-3} M(n, q, 1)+\left(\frac{3}{8}-n\right) C_{2 n-q-2} M(n, q, 2)\right] .
$$

Now subtracting the internal energies in two regions (eqs.(54) and (50)) and using eqs.(47) and (53), one obtains

$$
U_{2, n}^{(s)}-U_{2, n}^{(w)}=f(n)\left(\beta-\beta_{c}\right)^{2}+\ldots,
$$

where

$$
f(n)=\frac{(2 n-1)(4 n-3) b_{c}^{4 n-1}}{32 \sqrt{\pi} \beta_{c}^{4 n+1}}\left\{4 n T_{1}-\frac{4 n-3}{2(4 n-5)} T_{2}-\frac{4 n-3}{2} T_{4}\right\}
$$

in which

$$
T_{4}=C_{2 n-2} M(n, 0,2)+C_{2 n-3} M(n, 0,1)+2 \sum_{q=0} C_{2 n-q-4} M(n, q, 0) .
$$

Eq.(56) shows that all the $V=\operatorname{Tr}\left(A A^{\dagger}\right)^{n}$ gSCM has a third order phase transition near the critical point $\beta=\beta_{c}$. Note that $f(n)>0$ for all values of $n$, as we expected from the definitions of strong and weak regimes, and also the value of $f(n)$ for $n=2,3$, and 4 coincides with those in ref. [1]. 
As a final comment, it is interesting to compare the behavior of the solutions with respect to $n$. The only physical comparable quantity, in the case of third order phase transition, is the difference

$$
\frac{\partial C_{s}}{\partial T}-\frac{\partial C_{w}}{\partial T}=\beta^{2} \frac{\partial}{\partial \beta}\left(\beta^{2} \frac{\partial}{\partial \beta}\left(U^{(s)}-U^{(w)}\right)\right),
$$

where $C$ is the specific heat of the theory and $T=1 / \beta$ is temperature. Using (56), it is found

$$
\beta^{2} \frac{\partial}{\partial \beta}(\Delta C)_{n}=2 \beta_{c}^{4} f(n) .
$$

In Fig. (1), where we have plotted $\beta^{2} \frac{\partial}{\partial \beta}(\Delta C)_{n}$ vs $n$, one can see a saturating value 2 for this difference.

\section{References}

[1] M. Alimohammadi; Nucl. Phys. B565 (2000) 469.

[2] G. 't Hooft; Nucl. Phys. B72 (1974) 461.

[3] D. J. Gross and E. Witten; Phys. Rev. D21 (1980) 446.

[4] R. C. Brower and M. Nauenberg; Nucl. Phys. B180 (1981) 221.

[5] E. Brezin and D. J. Gross; Phys. Lett. B97 (1980) 120.

[6] R. C. Brower, P. Rossi and C. -I. Tan; Phys. Rev. D23 (1981) 942; D23 (1981) 953.

[7] D. Friedan; Commun. Math. Phys. 78 (1981) 353.

[8] M. Campostrini, P. Rossi, and E. Vicari, Phys. Rev. D52 (1995) 358.

[9] P. Rossi and C. -I. Tan; Phys. Rev. D51 (1995) 7159.

[10] R. C. Brower, M. Campostrini, K. Orginos, P. Rossi, C. -I. Tan, and E. Vicari; Phys. Rev. D53 (1996) 3230.

[11] E. Witten; Jour. Geom. Phys. 9 (1992) 303.

[12] M. R. Douglas, K. Li and M. Staudacher; Nucl. Phys. B240 (1994) 140;

O. Ganor, J. Sonnenschein, and S. Yankielowicz; Nucl. Phys. B434 (1995) 139.

[13] M. Khorrami and M. Alimohammadi; Mod. Phys. Lett. A12 (1997) 2265.

[14] M. Alimohammadi, M. Khorrami and, A. Aghamohammadi; Nucl. Phys. B510 (1998) 313. 
[15] M. Alimohammadi and A. Tofighi; Eur. Phys. Jour. C8 (1999) 711.

[16] D. Gakhov, "Boundary problems" , Russian edition (Nauka, 1975), A. C. Pipkin; "A course on integral equations" (Springer, Berlin, 1991). 


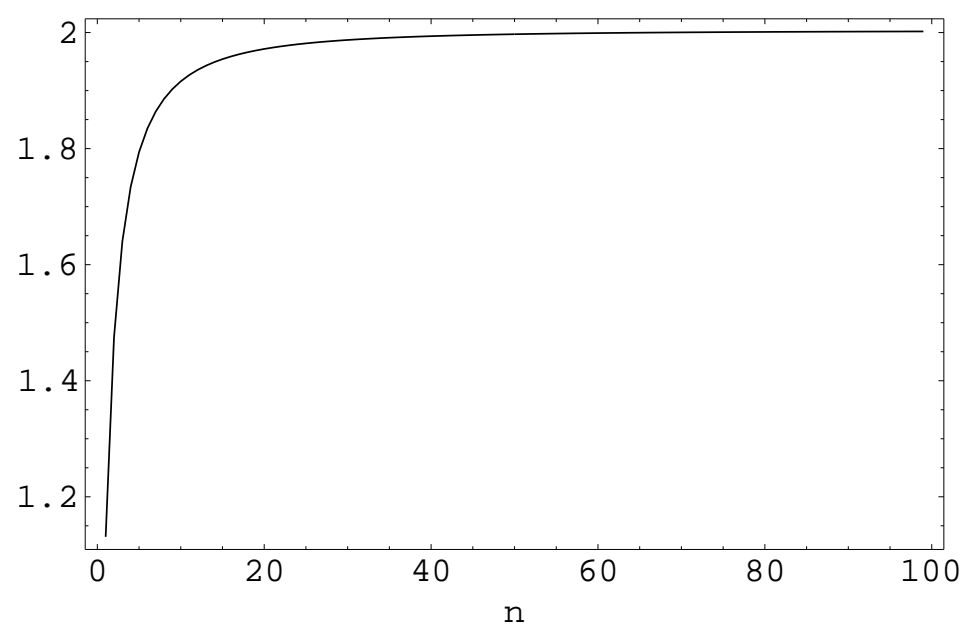

